\title{
Synergetic Interaction among Humans and Robotics by Proposing Communication Flow Methodology
}

\author{
R.K Seth \\ Professor \\ Department of Applied Science \\ Sri Sai University, HP (India)
}

\author{
Rimmy Chuchra \\ Assistant Professor \\ Department of Computer Science and Engg \\ Sri Sai Institute of Engg and \\ Technology,Manawala(Amritsar)
}

\begin{abstract}
This paper presents analysis of methodology proposed for mutual interaction among human and robotics following master slave relationship using three different types of interaction modes viz. co-existing, conditional and essential mode. These different modes of interaction during the performance of any desired task may include time for entering into the task, task processing time and task completion period. The collaboration between human and robotics leads to achieve a joint action for its utilization in developing projects in any industry and other daily life work. The technique designed in this paper helps human and robotics to handle queries and tasks using human robotics in such a way that robotics/human acts as an interface with the server system for the flow of communication data. The proposed procedure describes the way of humans interaction with the robotics and shows the complete communication flow among them either by using input in continuous (analog form) or in discontinuous (digital form). For providing more reliable and safer environment during communication, IVRE (Immersive virtual robotics environment) may be used with the help of virtual reality in virtual environment system.
\end{abstract}

\section{Keywords}

Human-Robotics, analog signal, digital signal.

\section{INTRODUCTION}

HRI helps to study the interactions between the humans and robotics [11]. Human-robotics interaction is a sub-field of human computer interaction whose discipline is concerned with the design, evaluation and implementation of interactive computing systems for human use [6].The basic goal of human robotics interaction is to define a general human model that could lead to principles and algorithms that allows more natural and effective interaction between human and robotics [4]. This field most commonly provide two types of interactions viz. remote interaction and proximate interaction for communication that are discussed below:

$>$ Remote Interactions: -In this type of interaction robotics are separated spatially or temporary as an example robotics can be operated with a remote under some specific area of range.

$>$ Proximate Interactions: - Both are co-located in the same room as an example human give command to robotics for performing any task.

For investigating human robotics communication people normally use cameras for visual reorganization or audio for sound reorganization as an example Infra-red sensors are used to capture the records from the natural touch [5] that may help us for better understanding of human motion [2]. The behavior of human robotics interaction is different in different environments as an example the use of commercial applications is totally different from the use of health care assistant applications [1]. They may use several types of interaction modes. Usually in our daily life, there are three types of interaction environments are to be considered viz. HRI, RHI, RRI that can be shown in fig. 1,2 \& 3 :

In fig. 1human acts as a master and robotics as a slave:
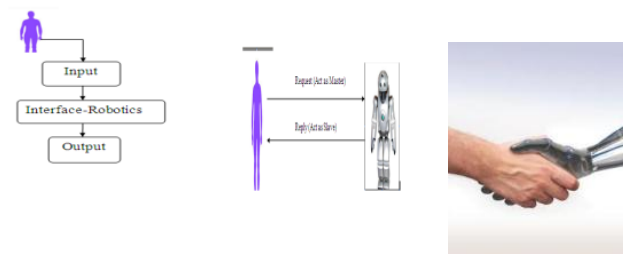

Fig 1 HRI [3, 4]: Human-robotics interaction

In fig. 2 robotics acts as a master and human as a slave:
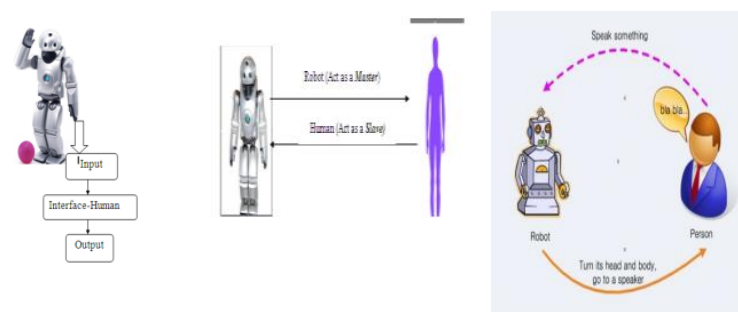

Fig 2 RHI: Robotics-Human Interaction

In fig. 3 shows robotics-robotics interaction where first robotics acts as a master and other robotics as a slave:

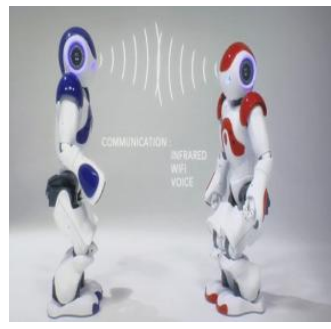

Fig 3 RRI: Robotics-Robotics Interaction

These three different types of interactions use 3 separate modes viz. co-existing interaction mode, conditional interaction mode and essential interaction mode. Each mode has their own function that can be shown with their schematic diagrams:

$>$ Co-existing interaction mode: In this mode robotics share environment with the humans that shows 
indirect participation as an example robotics helps for house Cleaning that can be shown in fig.4:

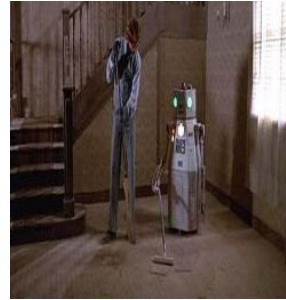

Fig 4 Robotics act as helper:House Cleaning

$>$ Conditional interaction mode: It's working is based on the instructions given by the humans(i.e. master) during performing any task as an example cooperation of robotics with surgeons during emergencies in OPD'S that can be shown in fig.5:

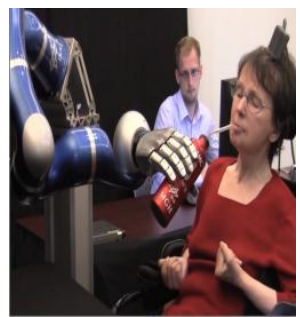

Fig 5 Robotics act asNurse:In hospitals helps to paitents for drinking water

$>$ Essential interaction mode: This mode shows the passive behavior of humans with robotics but performs essential role as an example instructions given by the team leader(i.e.-human who is master ) to the team worker (i.e. robotics who is slave) for completing the task of any project that can be shown in fig.6:

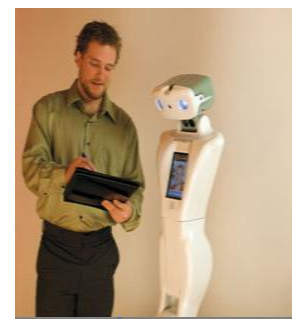

Fig 6 Robotics act as Team worker: Instructions given by Team Leader for amking project

These entire modes use component based software framework that allows for integration of operating system with independent components [7]. This artificial behavior of robotics has one most important distinct advantage that is they have capability to keep information private without any risk. And this intelligent behavior can be easily implemented by using different algorithms [9] where HRI is basically a highly inter-disciplinary field that brings together different methodologies and techniques as well [10].

This paper proposes a communication flow methodology procedure between human and robotics by considering two separate types of data input formats viz. analog form and digital form having two different environments viz. HRI environment \& RHI environment. The mutual interaction between human and robotics shows master slave relationship. The implementation of this proposed methodology in virtual environment provides a facility of safer \& reliable communication in future.

\section{REVIEW OF LITERATURE}

(Rimmy Chuchra \& Ramandeep Kaur et al Feb-2013) This paper discussed about the joint action taken by the humans and robotics for performing any task. Humans and robotics holds master/slave relationship where interface is act as a sandwich between the humans and robotics. The use of interface is to provide communication between human and robotics in natural way where human acts as a master and is responsible for giving instructions to robotics and robotics always acts as a slave that work as per instructions given by their master. The communication flow methodology between human and robotics can be shown in fig.7: [13]

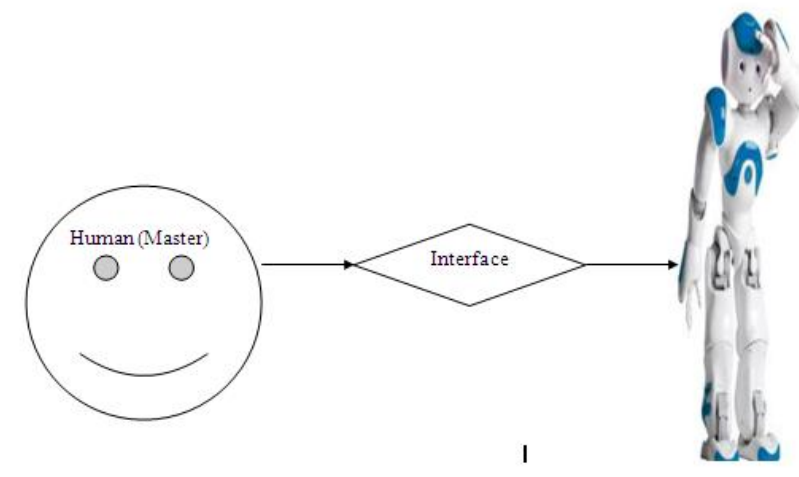

Fig 7 HRI: Shows Master/Slave Relationship

(Rimmy Chuchra \& R.K Seth et al May-June 2014): Authors had been proposed a new procedure termed as "Humanrobotics Interaction based analysis-Using data mining techniques" that shows how humans and robotics performed any task collectively by utilizing three different data mining techniques viz. classification, regression analysis and time series analysis. This designed methodology worked only with single type of data input format. It must be in discontinuous form (i.e. - in digital form). Theoretical and experimental based analysis investigated in this paper with significant results. [12]

\section{EXISTING PROCEDURE PLAN}

Step 1. When User Send Request: =Robot Mode Is ACTIVE.

\{Using classification technique, the active mode or de-active mode of the robot can be analyzed \}.MODE VALUE: = ACTIVE (indicates value 1). MODE VALUE: = DE-ACTIVE (indicates value 0 ).

Step 2. IF INPUT FORMAT CORRECT: =REQUEST PROCEDDING!

\{Using "Regression analysis technique" the relation between the previous tasks with the current task to be performed can easily be identified. It also gives the information between the independent and inter-related tasks that will be performed by the robot\}.

\section{Step 3. SET SESSION: = REQUEST COMPLETION}

\{Using "Time series Analysis technique" the completion time of task can be checked such that whether the task will be completed before the session expires\}.

Step 4. THEN REPEAT STEP 3 even "N" Number of tasks included. 


\subsection{Working}

The interface as shown in Figure 7 is a sandwich between human and robot for maintaining master-slave relationship where human act as a master and is responsible for performing number of tasks by Robot that acts like a slave and perform task as per instructions given by his master. At first, check the ROBOT is in ACTIVE MODE or DEACTIVE MODE. If the robot is in ACTIVE MODE then current will pass and shows value 1 otherwise signal disables and shows value 0 . After checking the status, in the second step, check the format of the input applied by the HUMAN (MASTER), whether it is in correct format or not. If input is in correct format, then REQUEST PROCEED otherwise generates a warning message. In the third step, in the request processing human set session, the task must be completed before session expires and if in case session expires, then request would be in pending mode and displays a warning message of request failure and THEN REPEAT step 3 even "N" number of tasks are to be performed[12].

\section{PROPOSED PROCEDURE PLAN}

Step 1. USER ENTER = ROBOT_ID.

Step 2. WHEN USER SEND REQUEST: = VERIFY INPUT TYPE FIRST

$$
\text { IF (INPUT: = DIGITAL FORM) }
$$

THEN REQUEST PROCEED! (BY USING ALGORITHM HRI BASED ANALYSIS Using DMT). [12]

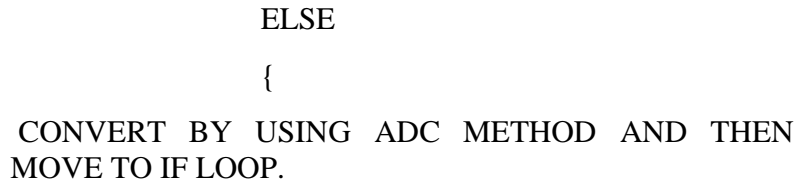

Step 3. END.

\subsection{Working}

The working of human and robotics in HRI environment holds following steps.

At first, in HRI environment, for accessing robotics, enter robotics_ID for unlock. After unlock the robotics, in second step, human who is actual master send request to robotics for performing any task then it first verifies the type of input applied viz. digital form or in analog form. If input is in digital form, then simply request will proceed by using algorithm termed as "HRI Based analysis-Using DMT" [3]. If input is in analog form, then at first it will be converted into digital form by using analog-to-digital converter method after that use the same procedure [3] and request will move to IF Loop. This procedure will use up to ' $\mathrm{N}$ ' numbers of tasks are available.

\section{COMMUNICATION FLOW PROCESS IN HRI ENVIRONMENT}

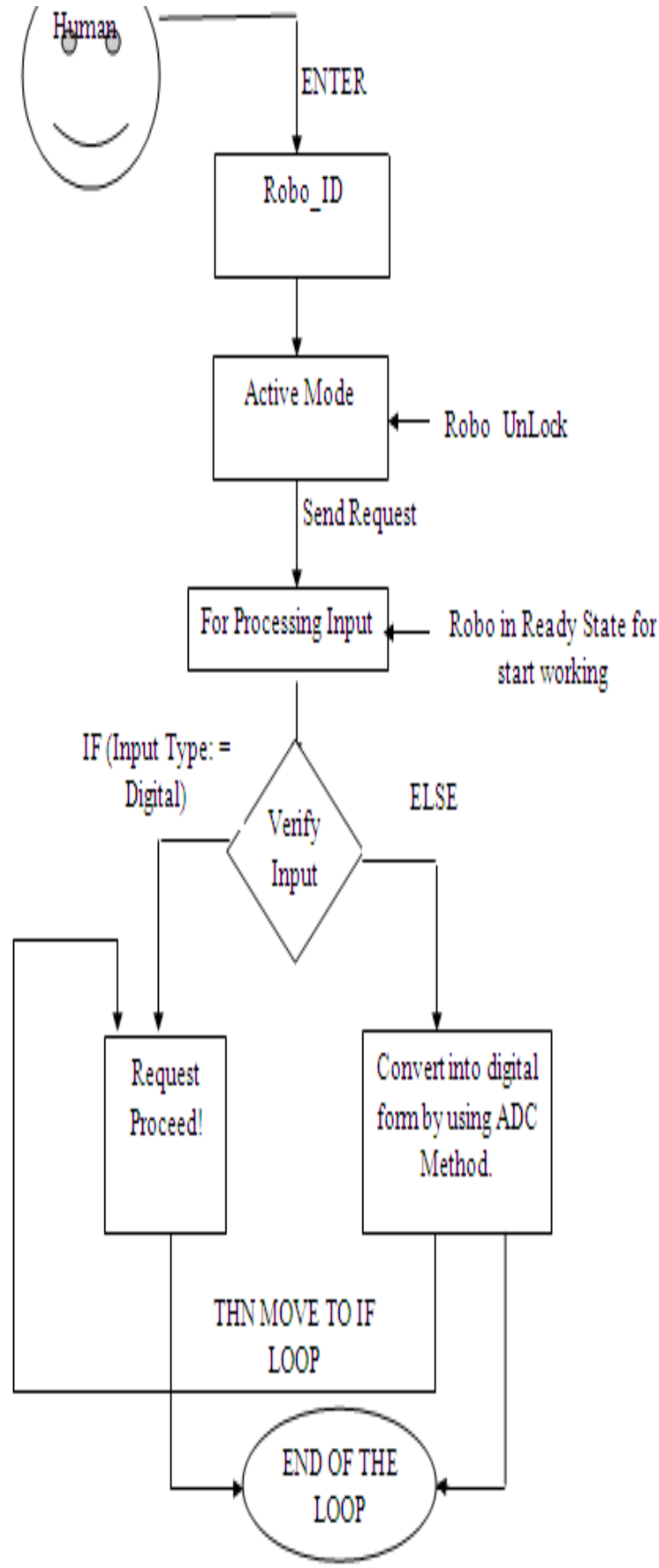

Fig 8 Working: In HRI Environment

\section{REAL LIFE APPLICATIONS FOR HRI ENVIRONMENT}

- Active role in Space over mars where humans control passively by using remote interactions that can be shown in fig.9: 


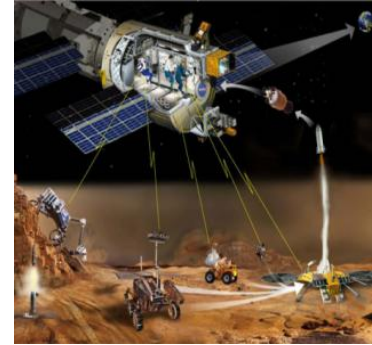

Fig 9 Communication of satellites with robotics: In Space

- Use of AVA Irobot in advance mobile platforms that uses multiple sensors for autonomous selfnavigation. These are basically telepresence machines \& look like televisions and can be shown in fig.10:

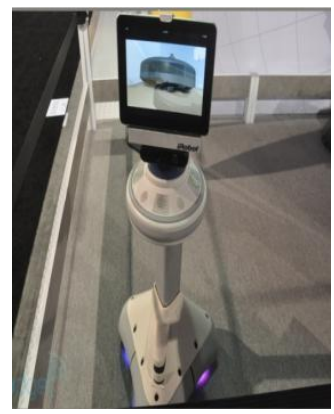

Fig 10 AVA Robotics: Used in mobile platforms

$>$ Domestic use for extending the use of social media in interpersonal interaction further to human-robot interaction (HRI) that can be shown in fig.11:

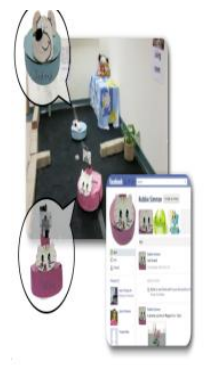

Fig 11 Domestic use of robotics: For entertainment purposes

\section{CONCLUSION}

This paper discussed about three separate type of interaction at separate modes and proposed a new communication flow procedure by considering two different data input formats viz. analog form and digital form. This procedure works with only HRI environment and carries master slave relationship. This proposed communication flow methodology as developed in this paper provides a more natural way utilizing interface. Instructions can be easily managed by robotics given by the master-human.

\section{FUTURE SCOPE}

In future, the synergetic interaction among human and robotics communication flow will be extended by designing some new mechanisms that helps to transfer human's natural skills to robotics by introducing the concept of technology transfer, so that machines will also behave naturally like humans as shown in fig. 12:

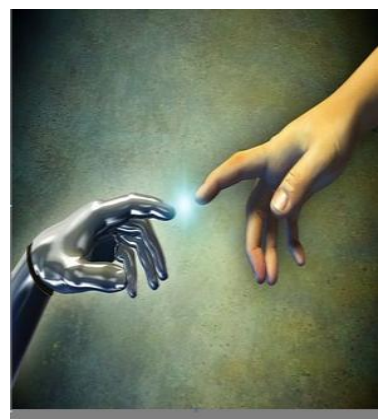

Fig 12 Transfer of natural Skills: Through Technology Transfer method

\section{REFERENCES}

[1] Cory D.Kidd, Human robotics interaction: Recent experiments and future work, IT Media lab.

[2] Hao Ding, Control robotics systems for safe interaction with human operators, proceeding of the $22^{\text {nd }}$ International joint conference on A.I, Institute of control \& system theory, University of Kassel, Germany.

[3] Michael A.GOODRICH \& Alan C.Schultz, Human robotics interaction: A survey Foundations \& Trends in human computer interaction, DOI: 10.15611110000005.

[4] Amit kumar, Rashmi Kumari \& Akash kumar Athgara, May 2014, A study on Human-Robotics interaction \& future applications", International journal of innovative Technology and adaptive management.

[5] Tamie salter,Renete boekhrost,Kerstin Dautenhalm, Learning about natural human robotics interaction styles",ELSEVIER:Adaptive system research group.

[6] Holly A.Yanco, Jill L-Drury, A Taxonomy for human robotics interaction, AAA International technical report FS-02-03.

[7] Kirchner, Nathan, David, singth \& Surya, Robotics Assist- A Platform for human robotics interactionResearch.

[8] Terrence Fong, Charles Thorpe -Verlag 2002. Collaboration, Dialogue \& Human robotics interaction, $10^{\text {th }}$ International symposium on robotics research, Lorne, Victoria, Australia, London: Springer.

[9] Algorithmic human robotics interaction workshop, Bielefeld, Germany.

[10] Illah Nourbaksh \& Reid Simmons.2014. Human robotics Interaction, Springer.

[11] robot.Kaist.ac. $\mathrm{Kr} /$ ?page-id=111.

[12] Rimmy Chuchra \& R.K Seth March-2014. Human robotics interaction based analysis-Using DMT, International Journal of Computer Science and Information Technology.

[13] Rimmy chuchra \& Ramandeep kaur, Feb 2013. Human robotics interaction with data mining techniques", International Journal of Emerging Technology And Advance Engg. 